\title{
Neuroimaging Correlates of Frontotemporal Dementia Associated with SQSTM1 Mutations
}

Elkin Luis $^{\mathrm{a}, \mathrm{b}}$, Alexandra Ortiz ${ }^{\mathrm{a}}$, Luis Eudave ${ }^{\mathrm{a}}$, Sara Ortega-Cubero $^{\mathrm{c}, \mathrm{d}}$, Barbara Borroni ${ }^{\mathrm{h}}$, Julie van der Zee ${ }^{\mathrm{f}, \mathrm{g}}$, Stefano Gazzina ${ }^{\mathrm{h}}$, Paola Caroppo ${ }^{\mathrm{i}, \mathrm{j}}$, Elisa Rubino M.A ${ }^{\mathrm{e}}$, Federico D’Agata ${ }^{\mathrm{e}}$, Isabelle Le Ber ${ }^{\mathrm{j}}$, Isabel Santana ${ }^{\mathrm{k}}$, Gil Cunha ${ }^{1}$, Maria R. Almeida ${ }^{\mathrm{m}}$, Claire Boutoleau-Bretonnière ${ }^{\mathrm{n}}$, Didier Hannequin $^{\mathrm{o}, \mathrm{p}, \mathrm{q}, \mathrm{r}}$, David Wallon ${ }^{\mathrm{p}, \mathrm{q}}$, Innocenzo Rainero ${ }^{\mathrm{s}}$, Daniela Galimberti ${ }^{\mathrm{t}}$,

Christine Van Broeckhoven ${ }^{\mathrm{f}, \mathrm{g}}$, Maria A. Pastor ${ }^{\mathrm{a}, \mathrm{b}, \mathrm{d}, \mathrm{u}}$ and Pau Pastor , $^{\mathrm{c}, \mathrm{v}, *}$

${ }^{a}$ Neuroimaging Laboratory, Division of Neurosciences, Center for Applied Medical Research (CIMA), University of Navarra, Pamplona, Spain

${ }^{\mathrm{b}}$ School of Education and Psychology, University of Navarra, Pamplona, Spain

${ }^{\mathrm{c}}$ Neurogenetics Laboratory, Division of Neurosciences, Center for Applied Medical Research, University of Navarra, Pamplona, Spain

${ }^{\mathrm{d}}$ CIBERNED, Centro de Investigación Biomédica en Red de Enfermedades Neurodegenerativas, Instituto de Salud Carlos III, Spain

e Università degli Studi di Torino, Department of Neuroscience "Rita Levi Montalcini”, University of Turin, Turin, Italy

${ }_{\mathrm{f}}^{\mathrm{f}}$ Neurodegenerative Brain Diseases Group, Department of Molecular Genetics, VIB, Antwerp, Belgium

${ }^{\mathrm{g}}$ Laboratory of Neurogenetics, Institute Born-Bunge, University of Antwerp, Antwerp, Belgium

${ }^{\mathrm{h}}$ Centre for Aging Brain and Neurodegenerative Disorders, Neurology Unit, University of Brescia, Brescia, Italy

${ }^{\mathrm{i} B e s t a}$ Neurological Institut, Milan, Italy

${ }^{\mathrm{j}}$ Institut du Cerveau et de la Moelle épinière (ICM), Sorbonne Universités, Université Pierre et Marie, Hôpital Pitié-Salpêtrière, Paris, France

${ }^{\mathrm{k}}$ Neurology Department, Centro Hospitalar e Universitário de Coimbra, Coimbra, Portugal and Faculty of Medicine, Coimbra University, Coimbra, Portugal

${ }^{1}$ Neurorradiology Department, Centro Hospitalar e Universitário de Coimbra, Coimbra, Portugal

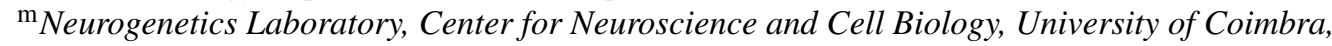

Coimbra, Portugal

${ }^{\mathrm{n}}$ Claire Boutoleau-Bretonnière: Department of Neurology, CMRR, Nantes University Hospital, Nantes, France

${ }^{\circ}$ Department of Genetics, Rouen University Hospital, Rouen, France

$\mathrm{p}^{\mathrm{p}}$ Inserm U1079, Rouen University, IRIB, Normandy University, Rouen, France

${ }^{\mathrm{q}}$ CNR-MAJ, Rouen University Hospital, Rouen, France

${ }^{\mathrm{r}}$ Department of Neurology, Rouen University Hospital, Rouen, France

${ }^{\mathrm{s}}$ Department of Neuroscience "Rita Levi Montalcini”, University of Torino, Turin, Italy

${ }^{\mathrm{t}}$ Department of Pathophysiology and Transplantation, University of Milan, Fondazione Ca' Granda,

IRCCS Ospedale Policlinico, Milan, Italy

\footnotetext{
${ }^{*}$ Correspondence to: Pau Pastor, MD, PhD, Department of Neurology, Hospital Universitari Mutua de Terrasa, Terrassa, Barcelona, Spain, Plaza Dr. Robert, 5, 08221-Terrasa, Spain. Tel.: +34 937365050; Fax: +34 937365059; E-mail: pastorpau@ gmail.com.
} 
u'Department of Neurology, Clínica Universidad de Navarra, University of Navarra School of Medicine, Pamplona, Spain

${ }^{\mathrm{v}}$ Memory and Movement Disorders Units, Department of Neurology, University Hospital Mutua de Terrassa, Terrassa, Barcelona, Spain

Accepted 31 March 2016

\begin{abstract}
.
Background: Frontotemporal lobar degeneration (FTLD) is a progressive dementia characterized by focal atrophy of frontal and/or temporal lobes caused by mutations in the gene coding for sequestosome 1 (SQSTM1), among other genes. Rare SQSTM1 gene mutations have been associated with Paget's disease of bone, amyotrophic lateral sclerosis, and, more recently, frontotemporal lobar degeneration (FTLD).

Objective: The aim of the study was to determine whether a characteristic pattern of grey and white matter loss is associated with SQSTM1 dysfunction.

Methods: We performed a voxel-based morphometry (VBM) study in FTD subjects carrying SQSTM1 pathogenic variants $(F T D / S Q S T M 1$ mutation carriers; $n=10)$, compared with FTD subjects not carrying SQSTM1 mutations (Sporadic FTD; $n=20$ ) and healthy controls with no SQSTM1 mutations (HC/SQSTM1 noncarriers; $n=20)$. The groups were matched according to current age, disease duration, and gender.

Results: After comparing FTD/SQSTM1 carriers with Sporadic FTD, a predominantly right cortical atrophy pattern was localized in the inferior frontal, medial orbitofrontal, precentral gyri, and the anterior insula. White matter atrophy was found in both medial and inferior frontal gyri, pallidum, and putamen. FTD/SQSTM1 carriers compared with HC/SQSTMI noncarriers showed atrophy at frontal, temporal, and parietal lobes of both hemispheres whereas the MRI pattern found in Sporadic FTD compared with controls was frontal and left temporal lobe atrophy, extending toward parietal and occipital lobes of both hemispheres.

Conclusions: These results suggest that fronto-orbito-insular regions including corticospinal projections as described in ALS are probably more susceptible to the damaging effect of SQSTM1 mutations delineating a specific gene-linked atrophy pattern.
\end{abstract}

Keywords: Dementia, frontotemporal dementia, SQSTM1 protein, voxel-based morphometry

\section{INTRODUCTION}

Frontotemporal lobar degeneration (FTLD) is a progressive dementia characterized by atrophy of frontal and/or temporal lobes resulting from different pathological processes, frequently linked to genetic mutations. FTLD is associated (15\%) with motor neuron disease (MND) or amyotrophic lateral sclerosis (ALS). A frequent cause of FTLD+/-MND is a $G 4 C 2$ repeat expansion in the chromosome 9 open reading frame 72 gene (C9ORF72; OMIM\#: 614260). Less frequently, FTLD is caused by mutations in other genes such as the microtubule-associated protein tau gene (MAPT; OMIM\#: 157140) [1], progranulin (GRN; OMIM\#: 138945) [2], valosin containing protein (VCP; OMIM\#: 601023), fused in sarcoma (FUS; OMIM\#: 137070), TAr DNA-binding pro- tein (TARDBP; OMIM\#: 605078) gene, and, very recently, in the gene coding for sequestosome 1 (SQSTM1; OMIM\#: 601530), which causes Paget disease of bone $[3,4,5,6,7]$.

The SQSTM1 gene is located in chromosome 5 and encodes for p62, a ubiquitin binding protein involved in protein phosphorylation and degradation, autophagosome formation, mediation of the NFkappaB activation pathway, and osteoclast growth factor regulation [8]. There are seven mutational studies reporting rare $S Q S T M 1$ variants associated with FTLD or ALS, suggesting that SQSTM1 has a significant role in both diseases $[9,10]$. Since there is no information on the regional brain effects of SQSTM1 variants in the literature, we hypothesized that VBM-studies in SQSTM1-carriers patients with FTLD can be useful for two reasons: first, in order to 
determine whether SQSTMImutations are associated with specific clinical FTLD phenotypes and secondly to identify whether the expression of SQSTM1 mutations in the brain have any significant regional differences when compared with non-carriers, thus reflecting an increased regional susceptibility.

\section{MATERIALS AND METHODS}

\section{Subject selection and comparison groups}

This is a multicenter retrospective and exploratory collaborative study in which all the members of the EU EOD consortium [10] and other authors of published studies analyzing the SQSTM1 gene $[10,11]$ were asked to provide available brain MRI scans from the subjects screened in previous studies whether they were FTD/SQSTM1 carriers or not. A subject was considered to be a FTD/SQSTM1 carrier when she/he fulfilled research criteria for FTLD [12] and carried a disease-segregating SQSTM1 genetic variant or, if DNA samples from relatives to demonstrate familial segregation were unavailable, their SQSTM1 variant, was only found in FTLD cases or the variants were more frequent among the cases [9, 11].

The T1-weighted images were obtained from five European research centers: the Center for Aging Brain and Neurodegenerative Disorders. Neurology Unit, University of Brescia, Brescia, Italy; University of Turin, Department of Neuroscience "Rita Levi Montalcini” Studi di Torino, Turin, Italy; Center de Recherche de I' Institut du Cerveau et de la Moelle Epinière, Hôpital de la Pitié-Salpêtrière - France; Centro Hospitalar e Universitário de Coimbra, Coimbra, Portugal and the Clínica Universidad de Navarra, Pamplona, Spain (Table 1). Seven MRI scans from the Sporadic FTD subjects were discarded because of poor image quality or movement artifacts. On the other hand, two FTD/SQSTM1 carrier patients were discarded, one because the images were not isotropic and another one because the patient carried two heterozygous FTLD-causing mutations: p.P392L SQSTM1 and the C9orf72 expansion.

Once we obtained the MRI scans, the group of FTD/SQSTM1 carrier subjects was double-matched with a group of Sporadic FTD/SQSTM1 subjects and a group of cognitively intact SQSTM1 noncarriers. The matching process was performed in order to have similar gender, age at onset, disease duration, and age at evaluation across groups (Table 2). Finally, except for a patient with semantic dementia, the FTD patients were matched across groups by their clinical FTD presentation. Sporadic FTD subjects did not carry mutations in the GRN, MAPT, or C9ORF72 genes.

\section{Demographic and clinical data and analysis}

Normality and variance homogeneity for the variables age at evaluation, Mini-Mental State Examination (MMSE), disease duration, and age at onset were included in the study and tested using the Shapiro-Wilk and Levene tests, respectively. Analysis of variance was applied to test the differences between the means of age at evaluation and MMSE variables among the three study groups. When only the two groups (FTD/SQSTM1 carriers and Sporadic $F T D$ ) were compared, a t-test was applied to evaluate age at onset and disease duration. FTD subtype and family history variables were compared using the exact $\chi^{2}$-test. The analysis was carried out using the program SPSS 15.0 software for Windows (SPSS Inc., Chicago, Illinois, USA). Bonferroni's correction was applied for multiple comparisons in all tests performed (Table 2).

Table 1

Number of participants in each group for center

\begin{tabular}{|c|c|c|c|}
\hline \multirow[t]{2}{*}{ Centers } & $\begin{array}{l}\text { FTD/SQSTM1 } \\
\text { carriers }\end{array}$ & $\begin{array}{l}\text { Sporadic } \\
\text { FTD }\end{array}$ & $\begin{array}{c}\text { HC/SQSTM1 } \\
\text { noncarriers }\end{array}$ \\
\hline & \multicolumn{3}{|c|}{ Number of participants } \\
\hline $\begin{array}{l}\text { Centre for Aging Brain and Neurodegenerative Disorders. Neurology } \\
\text { Unit, University of Brescia, Brescia, Italy }\end{array}$ & 2 & 8 & 0 \\
\hline $\begin{array}{l}\text { University of Turin, Department of Neuroscience "Rita Levi } \\
\text { Montalcini" degli Studi di Torino, Turin, Italy }\end{array}$ & 2 & 5 & 0 \\
\hline $\begin{array}{l}\text { Centre de Recherche de I' Institut du Cerveau et de la Moelle Epinière, } \\
\text { Hôpital de la Pitié-Salpêtrière - France }\end{array}$ & 0 & 5 & 0 \\
\hline Centro Hospitalar e Universitário de Coimbra, Coimbra, Portugal & 3 & 0 & 0 \\
\hline $\begin{array}{l}\text { Clínica Universidad de Navarra, School of Medicine in the Memory } \\
\text { Disorders Unit, Department of Neurology, Pamplona, Spain }\end{array}$ & 1 & 14 & 20 \\
\hline
\end{tabular}




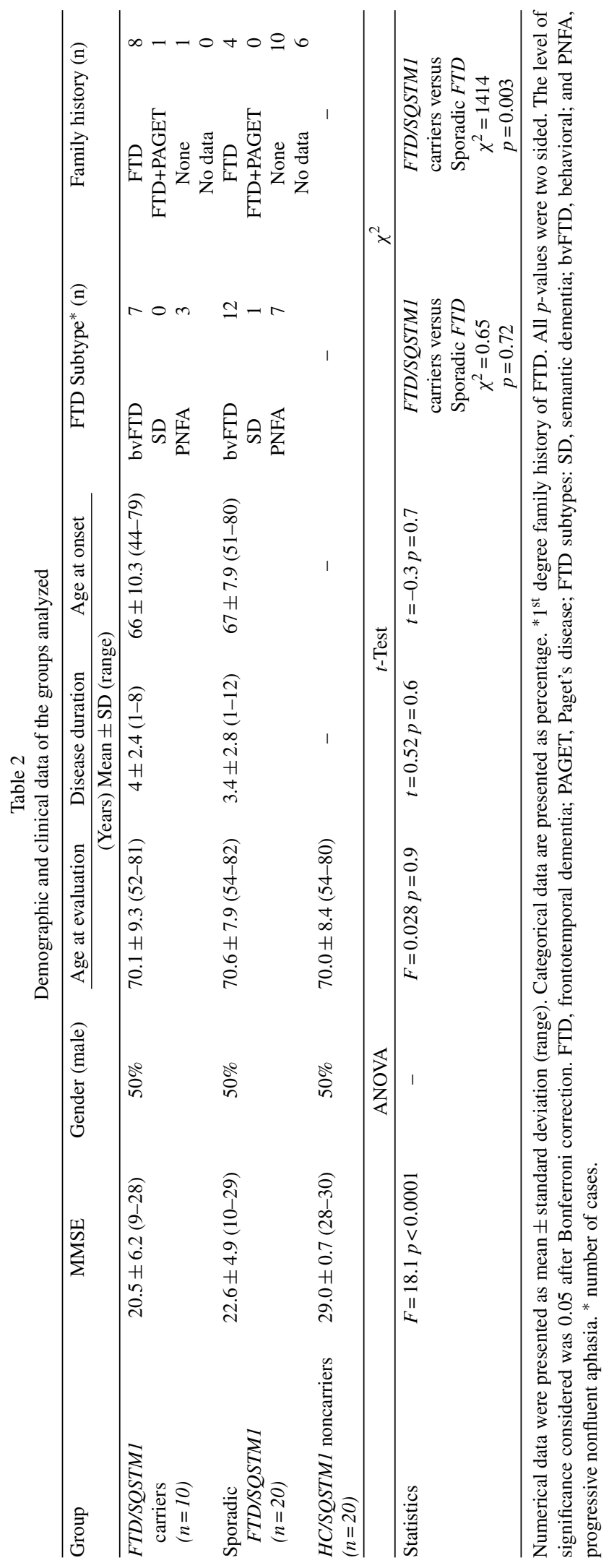



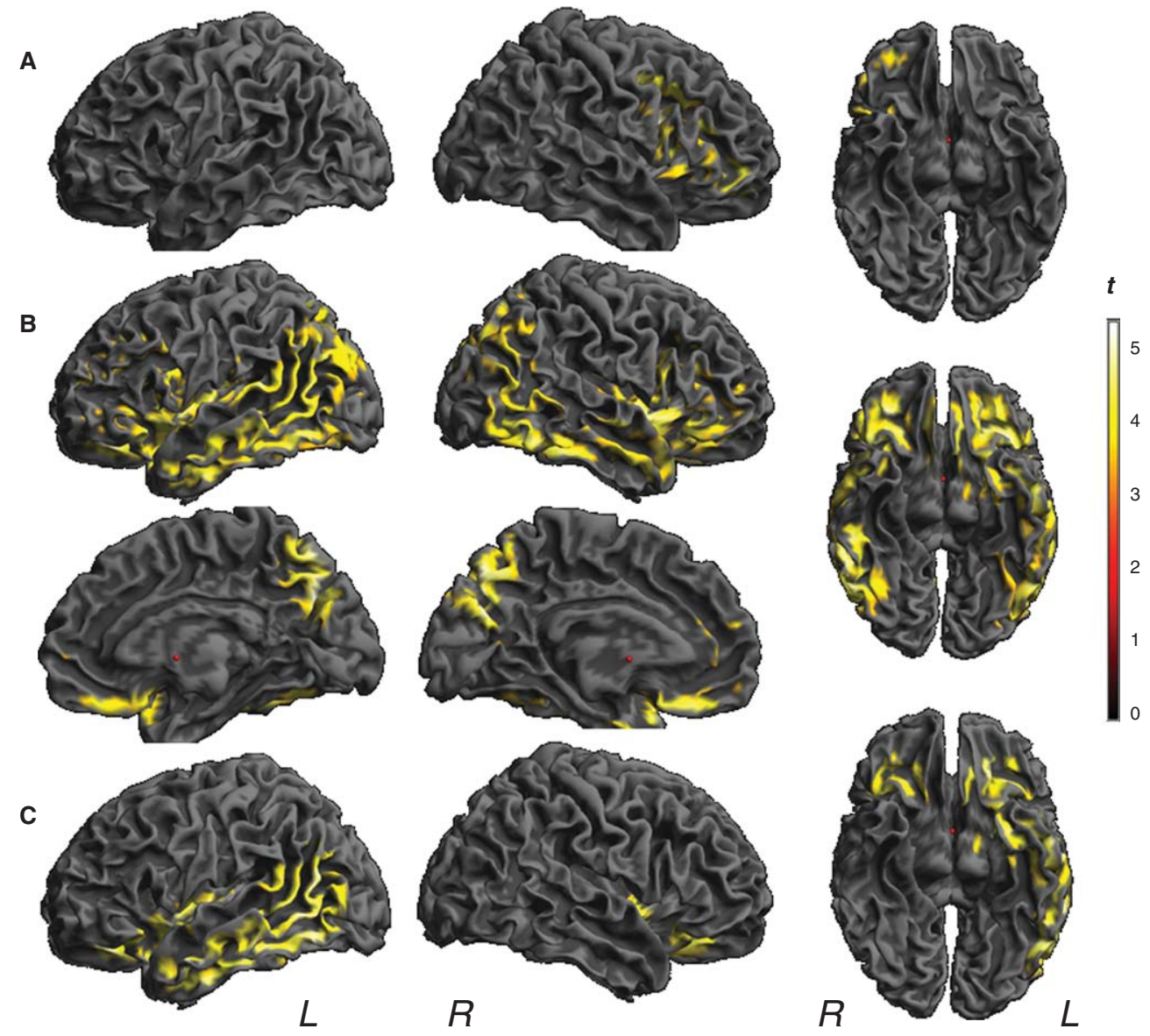

Fig. 1. Analysis displaying grey matter loss on the rendered surface of the brain in the following contrasts: A) Contrast FTD/SQSTM1 carriers versus Sporadic FTD (Threshold $p<0.05$. FWE cluster-wise $p<0.01$ ); B) FTD/SQSTM1 carriers versus HC/SQSTM1 noncarriers; and C) Sporadic FTD versus HC/SQSTM1 noncarriers (Threshold $p<0.05$. FWE cluster-wise $p<0.001$ ).

\section{Neuroimaging assessment}

\section{Data acquisition}

The T1-weighted images obtained from five centers used five different $1.5 \mathrm{~T}$ scanners. Three FTD/SQSTM1 carriers underwent their MRI scan in $1.5 \mathrm{~T}$ GE Medical Systems scanner at two different centers, 5 FTD/SQSTM1 carriers in 1.5-T Siemens Symphony scanner at two different centers, and 2 in 1.5-T Philips Medical Systems at only one center. The MRI scans from Sporadic FTD and 20 HC/SQSTMI subjects were acquired in 1.5-T Siemens Symphony scanners at two different centers. All T1-weighted images had a voxel size of $1 \times 1 \times 1 \mathrm{~mm}$.

\section{MRI pre-processing}

Determination of image quality followed the local internal protocol consisting of examination of the voxel size, the isotropic characteristic of the images and the movement artifacts. Secondly, after the preprocessing, we performed a covariance matrix that showed the covariance between volumes in each subject. These covariance values (Supplementary Figure 1) demonstrated the homogeneity of the sample.

We used the Statistical Parametric Mapping 8 software (SPM8; Wellcome Trust Centre for Neuroimaging; University College London, UK) implemented in Matlab 7.1 environment (MathWorks, Inc.) for image preprocessing. All images were transformed from the standard DICOM format (Digital Imaging and Communication in Medicine) to the NIfTI format (Neuroimaging Informatics Technology Initiative); the MNI localization of each image was manually rectified using the anterior and posterior commissures and anatomical references. First, we generated a custom template using the Diffeomorphic Anatomical Registration with the Exponentiated 
Lie Algebra algorithm tool (DARTEL) [13]. Subsequently, the images were processed using the New Segment tool for defining tissue probability maps through non-linear deformation and registration, in order to obtain grey matter (GM), white matter (WM) and cerebrospinal fluid images [13]. These individual GM and WM images were spatially normalized with the DARTEL- custom template made with the images of the population tested in this study. In this image processing method, we multiplied each GM and WM image spatially normalized by its total intracranial volume before and after normalization (modulation step). Finally, they were smoothed with a full-widthhalf-max (FWHM) 8-mm Gaussian kernel to increase the variation signal in the anatomical structure.

\section{Statistical group analysis}

Segmented images from FTD/SQSTM1 carriers, Sporadic FTD and HC/SQSTMI noncarriers were compared in a voxel-wise manner by performing two ANOVA analyses (one for GM and another for WM): these ANOVAs included a 'group' factor with three levels (FTD/SQSTM1 carriers, Sporadic FTD and HC/SQSTM1 noncarriers). Since the subjects were age- and gender-matched, the scanner type was included as covariate with the goal of eliminating its possible effect on the results. After determining a significant mean effect of the group factor, the following pair-wise SPM-t comparisons were carried out: FTD/SQSTM1 carriers versus Sporadic FTD noncarriers, FTD/SQSTM1 carriers versus HC/SQSTM1 noncarriers, and Sporadic FTD versus HC/SQSTM1 noncarriers.

The statistical significance of the contrasts of FTD/SQSTM1 carriers versus HC/SQSTM1 noncarriers and Sporadic FTD versus HC/SQSTM1 noncarriers was set up with a primary threshold of voxel-wise $p=0.001$, which yielded a cluster-extent based threshold of $k=41976$ and 2018 in $\operatorname{GM}(t=3.2)$ and $k 1022$ and 825 in WM $(t=3.2)$, respectively. For the contrast of FTD/SQSTM1 carriers versus Sporadic FTD noncarriers, a primary threshold of voxel-wise $p<0.01$ was set up due to low statistical power, which yielded a cluster-extent based thresholding of $k=6124$ in GM $(t=2.4)$ and 5590 in WM $(t=2.4)$. Finally, the threshold for the resulting clusters in each contrast was limited $p<0.05$ family-wise error (FWE) correction. The cluster-extent based thresholding was calculated with the Gaussian random field (GRF) in Statistical Parametric Mapping (SPM8) using the estimated intrinsic smoothness based on residual images. Tables were created using the SPM8 Anatomy toolbox version 1.5 [14], and anatomical areas reported were based on its V20 maps for GM. For WM evaluation we used a tractographybased atlas of human brain connections from the Natbrainlab website (http://www.natbrainlab.com/).

\section{RESULTS}

\section{Subjects available for the VBM analysis}

In this study, ten FTD/SQSTM1 carriers (median age $=70.1$; range $=52-81 ; 50 \%$ male) were compared with twenty Sporadic FTD (median age =70.6; range $=54-82 ; 50 \%$ male $)$ and twenty $H C / S Q S T M 1$ noncarriers $($ median age $=70.0 ;$ range $=54-80 ; 50 \%$ male) (Table 2). All groups were matched by gender, age at evaluation, and disease duration among patients. The FTD/SQSTM1 carrier group included two subjects with the SQSTM1 p.L238del variant and the other subjects carried one of the following SQSTM1 variants: p.P392L, p.P387L, pA33, E319K, P348L, P439L, T430P, and p.D329G (Table 1). With regard to the clinical subtypes of the FTD/SQSTM1 carriers, seven patients were diagnosed with behavioral FTD subtype (bvFTD) and three with progressive non-fluent aphasia (PNFA). In addition, one patient also had ALS, one Paget's disease, two presented parkinsonian signs and one psychotic symptoms. Among the Sporadic FTD, twelve patients had the bvFTD subtype, seven PNFA, and one semantic dementia (SD).

\section{Demographic and clinical analyses}

There were no significant differences in age at evaluation across the groups $(F=0.02, p=0.9)$. There were no differences between FTD/SQSTMI carriers and Sporadic FTD in the other demographic variables such as the age at onset $(t=-0.3, p=0.7)$, disease duration $(t=0.5, p=0.6)$, and FTD clinical subtype $\left(\chi^{2}=0.65, p=0.72\right)$. We found significant differences in MMSE (group factor $F=18.1, p<0.0001$ ) in FTD/SQSTM1 carriers versus HC/SQSTM1 noncarriers $(p<0.0001)$ and Sporadic FTD versus $H C / S Q S T M 1$ noncarriers comparisons $(p<0.0001)$. There were no MMSE score differences between FTD/SQSTM1 carriers and Sporadic FTD $(p=0.5)$. Finally, differences were also found in the frequency of family history of FTD and/or ALS, which was higher in the FTD/SQSTMI carriers $\left(\chi^{2}=1414\right.$, $p=0.003$ ) (Table 2). 
Table 3

Volumetric grey matter loss contrast between FTD/SQSTM1 carriers versus Sporadic FTD

\begin{tabular}{|c|c|c|c|c|c|c|c|c|}
\hline Side & Area & BA & $\mathrm{k}$ & p-value & $t$ & $\mathrm{x}$ & $\mathrm{y}$ & $\mathrm{Z}$ \\
\hline $\mathrm{R}$ & Inferior Frontal Gyrus p. Triangularis & 45 & 6124 & 0.01 & 3.78 & 42 & 11 & 24 \\
\hline \multirow[t]{2}{*}{$\mathrm{R}$} & Inferior Frontal Gyrus p. Opercularis & 44 & & & 3.74 & 41 & 11 & 22 \\
\hline & & 44 & & & 3.17 & 53 & 11 & 12 \\
\hline $\mathrm{R}$ & Inferior Frontal Gyrus p. Orbitalis & 44 & & & 3.27 & 45 & 41 & -3 \\
\hline \multirow[t]{2}{*}{$\mathrm{R}$} & Precentral Gyrus & 6 & & & 3.20 & 45 & -2 & 45 \\
\hline & & & & & 3.00 & 33 & -1 & 48 \\
\hline \multirow[t]{2}{*}{$\mathrm{R}$} & Middle Frontal Gyrus & 8 & & & 3.46 & 38 & 14 & 42 \\
\hline & & & & & 3.37 & 35 & 23 & 37 \\
\hline $\mathrm{R}$ & Middle Orbital Gyrus & $10,11,46$ & & & 3.32 & 35 & 53 & -3 \\
\hline $\mathrm{R}$ & Insula Lobe & 44 & & & 3.37 & 41 & 12 & 3 \\
\hline
\end{tabular}

Statistically significant white matter loss; Side: laterality; BA: Brodmann Areas; k: number of voxels per cluster. $p<0.05$ FWE cluster-wise corrected $p=0.01 ; t$ : T-test score; x.y.z : MNI coordinates.

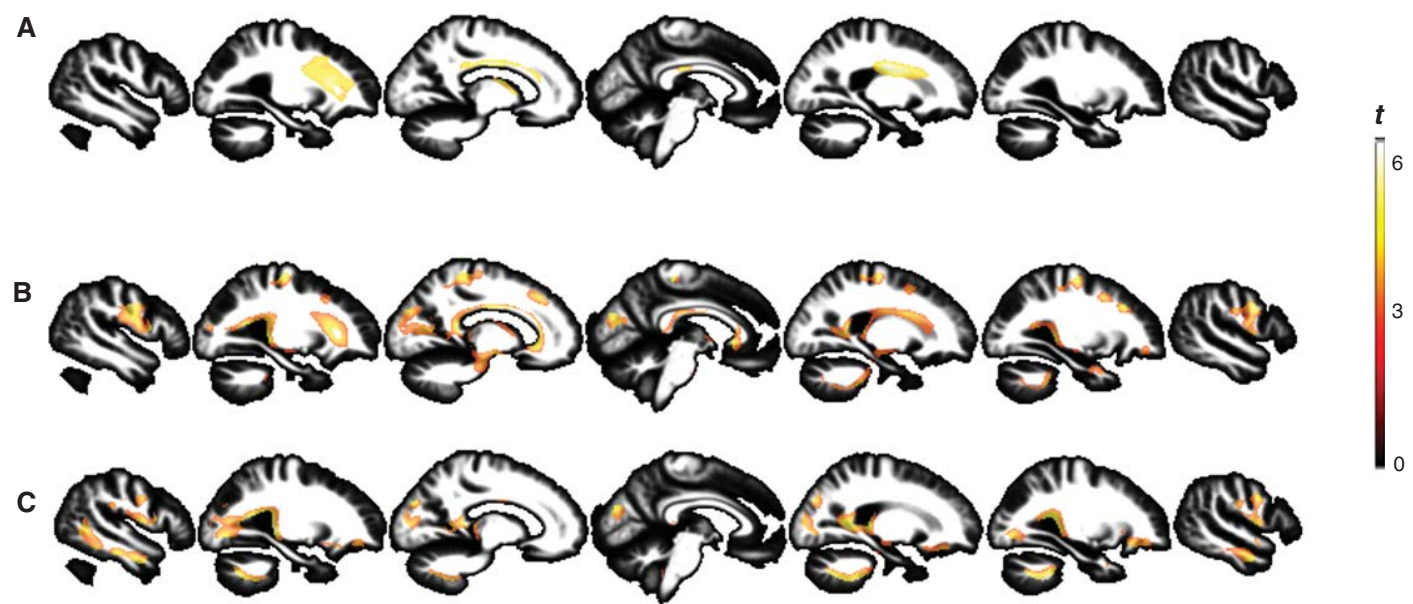

Fig. 2. Analysis displaying white matter loss on the rendered surface of the brain in the following contrasts: A) Contrast FTD/SQSTM1 carriers versus Sporadic FTD (Threshold $p<0.05$. FWE cluster-wise $p<0.01$ ); B) FTD/SQSTM1 carriers versus HC/SQSTM1 noncarriers; and C) Sporadic FTD versus HC/SQSTM1 noncarriers (Threshold $p<0.05$. FWE cluster-wise $p<0.001$ ).

\section{Grey matter VBM analysis}

ANOVA analyses of GM data showed a significant main effect of the Group factor $(F=8.057$, $p<0.0001)$.

\section{Comparison between FTD/SQSTM1 carriers versus Sporadic FTD groups}

On a direct comparison, the FTD/SQSTM1 carriers had smaller GM volumes than the Sporadic FTD/SQSTM1 predominantly in the right hemisphere, including inferior and middle frontal gyrus, middle orbital gyrus, insula, and precentral gyrus (Fig. 1A, Table 3, and Supplementary Table 1). These results reflected a grey matter loss pattern among the FTD/SQSTMI carriers with impairment of right prefrontal-orbital-insular regions with relative preservation of the temporal lobes. In short,
FTD/SQSTM1 carriers' atrophy pattern was specific to the prefrontal cortex, involving premotor and primary motor regions (Fig. 1 and Table 3). The prefrontal right atrophic clusters were extended bilaterally when this same contrast was thresholded to $t=2.4, \quad p<0.01$ uncorrected (Supplementary Figure 2).

\section{Comparison between FTD/SQSTM1 carriers versus HC/SQSTM1 noncarriers}

The 10 FTD/SQSTM1 carriers were compared with $20 \mathrm{HC} / S Q S T M 1$ noncarriers and showed severe GM volume loss ( $p<0.05 \mathrm{FWE}$ cluster-wise corrected) in the following regions: insula, inferior temporal gyrus, precuneus, and inferior frontal gyrus of both hemispheres, plus the right temporal lobe and cuneus and middle orbital gyrus of the left hemisphere (Fig. 1B, Supplementary Table 1). 


\section{Comparison between Sporadic FTD versus} HC/SQSTM1 noncarriers

We observed widespread GM loss in the 20 Sporadic FTD compared with the 20 HC/SQSTM1 noncarriers $(p<0.05$ FWE cluster-wise corrected) with a predominant distribution in temporal and frontal lobes. This pattern was consistent with the results of previous VBM studies performed in FTLDcontrol series where the underlying genetic causes were not known [15]. The temporal atrophic clusters were located in the left middle temporal gyrus, temporal pole and superior temporal gyrus and in frontal regions. The brain atrophy was specifically intense at the insula and inferior and middle orbital gyrus (Fig. 1C and Supplementary Table 2).

\section{White matter VBM analysis}

ANOVA analyses of WM data showed a significant main effect of the Group factor $(F=8.057$, $p<0.0001$ ).

\section{Comparison between FTD/SQSTM1 carriers versus Sporadic FTD/ SQSTM1}

The contrast between WM brain volume FTD/ SQSTM1 carriers versus Sporadic FTD/ SQSTM1 resulted in significant differences in regions underlying both medial and left inferior frontal gyrus, triangular area, and in neighboring regions to left pallidum globe and putamen (Fig. 2A and Supplementary Table 3).

\section{Comparison between FTD/SQSTM1 carriers versus HC/SQSTMI noncarriers}

This contrast showed WM volume loss $(p<0.05$ FWE cluster-wise corrected) in underlying regions to the inferior frontal gyrus, primary motor cortex, precuneus, superior occipital gyrus and particularly along the borders of the insula, amygdala, and hippocampus (Fig. 2B and Supplementary Table 4).

\section{Comparison between Sporadic FTD versus HC/SQSTM1 noncarriers}

Finally, Sporadic FTD versus HC/SQSTM1 noncarriers contrast $(p<0.05$ FWE cluster-wise corrected) showed an atrophy pattern in corticoponto-cerebellar regions such as the superior and inferior cerebellar peduncles. We also found WM vol- ume loss in occipital and frontal regions, the middle temporal lobe and hippocampus (Fig. 2C and Supplementary Table 5).

\section{DISCUSSION}

The present study is the result of a multicenter collaborative effort provided by the EU EOD consortium [10] where members were invited along with researchers involved in the other studies that have screened the SQSTM1 gene in FTLD subjects [10, 16] to collect available brain MRI scans from subjects with and without SQSTM1 mutations. The aim of our study was to analyze whether SQSTM1 pathogenic variants lead to a specific regional neurodegenerative pattern. For this purpose we used VBM MRI, which we have used successfully in the past to identify regional brain atrophy associated with other genetic variants, even in relatively small samples [17, 18]. In these studies, we reported that morphometric neuroimaging studies could identify brain regions sensitive to the damaging effects of specific genetic variants $[15,17,18,19]$.

In the present study, we found brain volume differences between $F T D / S Q S T M 1$ carriers versus Sporadic FTD, where the GM loss in FTD/SQSTM1 carrier group followed an asymmetric right pattern, with involvement of fronto-orbital regions, right inferior frontal gyrus (pars triangularis, pars opercularis and pars orbitalis), insula lobe, premotor and motor areas (Fig. 1 and Table 3). The right inferior frontal gyrus has been associated with cognitive emotion regulation and executive functions, especially for salience of external clues, regardless of whether that detection is followed by the inhibition of a motor response, the generation of a motor response, or no external response at all [20]. The right anterior insula belongs to the ventral attention system with connectivity with frontal lobe regions implicated in goal-directed behavior $[21,22]$. On the other hand, the premotor cortex is involved in visuomotor transformation tasks, spatial orientation, learning, and cognitive functions $[23,24]$. The pattern of atrophy found in FTD/SQSTM1 carriers involved the impairment not only of the precentral cortex and corticospinal tracks but also premotor and prefrontal cortex, which are also impaired in human motorneuron disease [25].

Mutations in SQSTM1 gene were first described to cause Paget's disease [7] and years later familial ALS [9]. In fact, the substantial loss of brain volume in pre-motor cortical areas and in the cortico-spinal 
tract observed in our FTD/SQSTM1 carriers group was not found in the Sporadic FTD when compared to the control group. We suggest that this pattern can be due to a subclinical impairment of the premotor cortical areas and the cortico-spinal tract in the FTD/SQSTM1 carriers group, since SQSTM1 is also a disease-causing ALS gene. In a VBM study of ALS with behavioral symptoms [26], the ALS patients with apathy, depression, and anxiety showed an atrophy profile very similar to that found in the present study after comparing the FTD/SQSTMI carriers with Sporadic FTD, comprising premotor and motor areas and corticospinal tracts. These results reinforce the idea that there could be an important association between mutations in the SQSTM1 gene and the FTD-MND spectrum [9-11]. We want to highlight the point that our study collected brain MRI scans from FTD subjects with different mutations in diverse SQSTM1 gene domains that can result in variable structural and functional abnormalities of the protein leading to different disease phenotypes [10] and, subsequently, to specific patterns of brain atrophy. However, since mutations in SQSTMI gene are so rare, it was not possible to assess the individual morphological brain effect for each of the SQSTM1 variants.

To the best of our knowledge, there are no VBM studies in patients with FTD and SQSTM1 mutations, though one preliminary study measuring gray matter density in twelve patients with Paget's disease of bone and SQSTM1 mutations without FTD described a predominantly left frontotemporal atrophy pattern among patients [27].

Among the previous literature on VMB MRI analysis in other FTLD mutated genes [28, 29], Rohrer et al. [29] performed a VMB MRI analysis of MAPT and GRN mutations in FTLD carriers compared with healthy controls and found among $G R N$ mutation carriers a brain atrophy pattern with areas in common with that which we identified in our study, which included the orbitofrontal cortex and ventral insula, with marked extra atrophy of the anterior and medial temporal lobes and cingular cortex with a strong asymmetric pattern. However, we have to consider that they used a different methodology that enhances the GM loss. By contrary, the VBM pattern found among MAPT carriers was predominantly temporal and symmetrical. On the other hand, our VBM atrophy pattern (FTD/SQSTM1 carriers versus Sporadic $F T D$ ) was quite different from the one found in another VBM study of FTLD GRN carriers compared to healthy controls, which showed greater loss in posterior temporal and parietal lobes in this group [27].

These authors performed an MRI analysis of three groups of subjects with GRN and MAPT mutations as well as C9ORF72 expansion carriers [30] describing prominent atrophy of the anteromedial temporal lobes in the MAPT group, in the inferior temporal and parietal lobes in the $G R N$ group, and in posterior cerebral regions including the cerebellum in the C9ORF72 group. Interestingly, the brain atrophy pattern described for the Sporadic FTD group (35\% of them diagnosed with FTD-ALS) was more consistent with the atrophy pattern that we found in the FTD/SQSTM1 carriers versus Sporadic FTD comparison.

In another VBM MRI study comparing C9ORF72 expansion carriers and noncarriers, a thalamic atrophy and cerebellar volume loss in C9ORF72 expansion carriers was found [31]. In fact, the brain atrophy profile was completely different from the one found in our FTLD SQSTM1 carriers' group which encompassed the inferior frontal-orbital regions, right IFG: pars triangularis, pars opercularis and pars orbitalis, insula lobe, premotor, and motor areas. These results can be influenced by a higher frequency of the PNFA subtype in our patients' group.

All the data derived from the VBM MRI studies in subjects with FTD-causing mutations highlight the fact that brain atrophy patterns can diverge according to the mutated gene, probably as a result of differences in mRNA expression and/or differential regional brain susceptibilities.

Although, we have previously identified patterns of regional brain atrophy associated with other genetic variants with similar study designs to the present one $[15,17,18]$, our study still has several limitations: the number of subjects is low, but this was expected considering the low frequency of SQSTM1 mutations among FTD patients. In addition, the distribution of the three populations studied (FTD/SQSTM1 carriers, Sporadic FTD and HC/SQSTM1 noncarriers) was not homogeneous among centers. This methodological imbalance, in spite of the common quality control, can introduce a bias, which was perhaps not totally corrected when introduced as a covariate in the analyses.

\section{CONCLUSIONS}

In summary, our results reflect that FTD/SQSTM1 carriers show a right atrophy pattern in prefrontal- 
orbital-insular regions with relative preservation of the temporal lobes different from that observed in Sporadic FTD. The WM atrophy was present in regions underlying the right medial frontal gyrus and left medial and inferior frontal gyrus and close to the left pallidum and putamen. However, our study is retrospective and exploratory and has a number of limitations (small sample size, multicenter study with different MRI scanners, and absence of DTI data). To better define the specificity of these patterns, larger cross-sectional and longitudinal imaging studies will be needed.

\section{ACKNOWLEDGMENTS}

We thank all participating subjects and their relatives for their contribution to the study. This work was supported by grants to P. Pastor from the Department of Health of the Government of Navarra, Spain (refs.13085 and 3/2008) and from the UTE project FIMA, Spain to P. P; E. O. Luis is supported by an Education Department grant form Government of Navarra (2011-2014). L. E. is supported by a grant from University of Navarra. Genotyping for the SQSTM1 variants was performed by the European Early-Onset (EU EOD) consortium and supported by an International Coordination Action grant- from the Research Foundation Flanders (FWO) to C. Van Broeckhoven.

Authors' disclosures available online (http://j-alz. com/manuscript-disclosures/16-0006r2).

\section{SUPPLEMENTARY MATERIAL}

The supplementary material is available in the electronic version of this article: http://dx.doi.org/ 10.3233/JAD-160006.

\section{REFERENCES}

[1] Hutton M, Lendon CL, Rizzu P, Baker M, Froelich S, Houlden H, Pickering-Brown S, Chakraverty S, Isaacs A, Grover A, Hackett J, Adamson J, Lincoln S, Dickson D, Davies P, Petersen RC, Stevens M, de Graaff E, Wauters E, van Baren J, Hillebrand M, Joosse M, Kwon JM, Nowotny P, Che LK, Norton J, Morris JC, Reed LA, Trojanowski J, Basun H, Lannfelt L, Neystat M, Fahn S, Dark F, Tannenberg T, Dodd PR, Hayward N, Kwok JB, Schofield PR, Andreadis A, Snowden J, Craufurd D, Neary D, Owen F, Oostra BA, Hardy J, Goate A, van Swieten J, Mann D, Lynch T, Heutink $\mathrm{P}$ (1998) Association of missense and 5'-splice-site mutations in tau with the inherited dementia FTDP-17. Nature 393, 702-705.
[2] Cruts M, Gijselinck I, van der Zee J, Engelborghs S, Wils H, Pirici D, Rademakers R, Vandenberghe R, Dermaut B, Martin JJ, van Duijn C, Peeters K, Sciot R, Santens P, De Pooter T, Mattheijssens M, Van den Broeck M, Cuijt I, Vennekens K, De Deyn PP, Kumar-Singh S, Van Broeckhoven C (2006) Null mutations in progranulin cause ubiquitin-positive frontotemporal dementia linked to chromosome $17 \mathrm{q} 21$. Nature 442, 920-924.

[3] Synofzik M, Born C, Rominger A, Lummel N, Schols L, Biskup S, Schule C, Grasshoff U, Klopstock T, Adamczyk C (2014) Targeted high-throughput sequencing identifies a TARDBP mutation as a cause of early-onset FTD without motor neuron disease. Neurobiol Aging 35, 1212.e11212.e5.

[4] Synofzik M, Maetzler W, Grehl T, Prudlo J, Vom Hagen JM, Haack T, Rebassoo P, Munz M, Schols L, Biskup S (2012) Screening in ALS and FTD patients reveals 3 novel UBQLN2 mutations outside the PXX domain and a pure FTD phenotype. Neurobiol Aging 33, 2949.e13-2949.e17.

[5] Van Langenhove T, van der Zee J, Van Broeckhoven C (2012) The molecular basis of the frontotemporal lobar degeneration-amyotrophic lateral sclerosis spectrum. Ann Med 44, 817-828.

[6] Dobson-Stone C, Hallupp M, Loy CT, Thompson EM, Haan E, Sue CM, Panegyres PK, Razquin C, Seijo-Martinez M, Rene R, Gascon J, Campdelacreu J, Schmoll B, Volk AE, Brooks WS, Schofield PR, Pastor P, Kwok JB (2013) C9ORF72 repeat expansion in Australian and Spanish frontotemporal dementia patients. PLoS One 8, e56899.

[7] Laurin N, Brown JP, Morissette J, Raymond V (2002) Recurrent mutation of the gene encoding sequestosome 1 (SQSTM1/p62) in Paget disease of bone. Am J Hum Genet 70, $1582-1588$.

[8] Rea SL, Majcher V, Searle MS, Layfield R (2014) SQSTM1 mutations-bridging Paget disease of bone and ALS/FTLD. Exp Cell Res 325, 27-37.

[9] Fecto F, Yan J, Vemula SP, Liu E, Yang Y, Chen W, Zheng JG, Shi Y, Siddique N, Arrat H, Donkervoort S, AjroudDriss S, Sufit RL, Heller SL, Deng HX, Siddique T (2011) SQSTM1 mutations in familial and sporadic amyotrophic lateral sclerosis. Arch Neurol 68, 1440-1446.

[10] Van der Zee J, Van Langenhove T, Kovacs GG, Dillen L, Deschamps W, Engelborghs S, Matej R, Vandenbulcke M, Sieben A, Dermaut B, Smets K, Van Damme P, Merlin C, Laureys A, Van Den Broeck M, Mattheijssens M, Peeters K, Benussi L, Binetti G, Ghidoni R, Borroni B, Padovani A, Archetti S, Pastor P, Razquin C, OrtegaCubero S, Hernandez I, Boada M, Ruiz A, de Mendonca A, Miltenberger-Miltenyi G, do Couto FS, Sorbi S, Nacmias B, Bagnoli S, Graff C, Chiang HH, Thonberg H, Perneczky R, Diehl-Schmid J, Alexopoulos P, Frisoni GB, Bonvicini C, Synofzik M, Maetzler W, vom Hagen JM, Schols L, Haack TB, Strom TM, Prokisch H, Dols-Icardo O, Clarimon J, Lleo A, Santana I, Almeida MR, Santiago B, Heneka MT, Jessen F, Ramirez A, Sanchez-Valle R, Llado A, Gelpi E, Sarafov S, Tournev I, Jordanova A, Parobkova E, Fabrizi GM, Testi S, Salmon E, Strobel T, Santens P, Robberecht W, De Jonghe P, Martin JJ, Cras P, Vandenberghe R, De Deyn PP, Cruts M, Sleegers K, Van Broeckhoven C (2014) Rare mutations in SQSTM1 modify susceptibility to frontotemporal lobar degeneration. Acta Neuropathol 128, 397-410.

[11] Rubino E, Rainero I, Chio A, Rogaeva E, Galimberti D, Fenoglio P, Grinberg Y, Isaia G, Calvo A, Gentile S, Bruni AC, St George-Hyslop PH, Scarpini E, Gallone S, Pinessi L, TODEM Study Group (2012) SQSTM1 mutations in 
frontotemporal lobar degeneration and amyotrophic lateral sclerosis. Neurology 79, 1556-1562.

[12] Neary D, Snowden JS, Gustafson L, Passant U, Stuss D, Black S, Freedman M, Kertesz A, Robert PH, Albert M, Boone K, Miller BL, Cummings J, Benson DF (1998) Frontotemporal lobar degeneration: A consensus on clinical diagnostic criteria. Neurology 51, 1546-1554.

[13] Ashburner J, Friston KJ (2005) Unified segmentation. Neuroimage 26, 839-851.

[14] Eickhoff SB, Stephan KE, Mohlberg H, Grefkes C, Fink GR, Amunts K, Zilles K (2005) A new SPM toolbox for combining probabilistic cytoarchitectonic maps and functional imaging data. Neuroimage 25, 1325-1335.

[15] Zhang Y, Tartaglia MC, Schuff N, Chiang GC, Ching C, Rosen HJ, Gorno-Tempini ML, Miller BL, Weiner MW (2013) MRI signatures of brain macrostructural atrophy and microstructural degradation in frontotemporal lobar degeneration subtypes. J Alzheimers Dis 33, 431-444.

[16] Le Ber I, Camuzat A, Guerreiro R, Bouya-Ahmed K, Bras J, Nicolas G, Gabelle A, Didic M, De Septenville A, Millecamps S, Lenglet T, Latouche M, Kabashi E, Campion D, Hannequin D, Hardy J, Brice A, French Clinical and Genetic Research Network on FTD/FTD-ALS (2013) SQSTM1 mutations in French patients with frontotemporal dementia or frontotemporal dementia with amyotrophic lateral sclerosis. JAMA Neurol 70, 1403-1410.

[17] Luis EO, Ortega-Cubero S, Lamet I, Razquin C, Cruchaga C, Benitez BA, Lorenzo E, Irigoyen J, Alzheimer's Disease Neuroimaging Initiative (ADNI), Pastor MA, Pastor P (2014) Frontobasal gray matter loss is associated with the TREM2 p.R47H variant. Neurobiol Aging 35, 2681-2690.

[18] Goni J, Cervantes S, Arrondo G, Lamet I, Pastor P, Pastor MA (2013) Selective brain gray matter atrophy associated with APOE epsilon4 and MAPT H1 in subjects with mild cognitive impairment. J Alzheimers Dis 33, 1009-1019.

[19] Snowden J, Neary D, Mann D (2007) Frontotemporal lobar degeneration: Clinical and pathological relationships. Acta Neuropathol 114, 31-38.

[20] Kohn N, Eickhoff SB, Scheller M, Laird AR, Fox PT, Habel U (2014) Neural network of cognitive emotion regulationan ALE meta-analysis and MACM analysis. Neuroimage 87, 345-355.

[21] Fox MD, Snyder AZ, Vincent JL, Corbetta M, Van Essen DC, Raichle ME (2005) The human brain is intrinsically organized into dynamic, anticorrelated functional networks. Proc Natl Acad Sci U S A 102, 9673-9678.
[22] Seeley WW, Menon V, Schatzberg AF, Keller J, Glover GH, Kenna H, Reiss AL, Greicius MD (2007) Dissociable intrinsic connectivity networks for salience processing and executive control. J Neurosci 27, 2349-2356.

[23] Rizzolatti G, Luppino G (2001) The cortical motor system. Neuron 31, 889-901.

[24] Picard N, Strick PL (2001) Imaging the premotor areas. Curr Opin Neurobiol 11, 663-672.

[25] Chio A, Pagani M, Agosta F, Calvo A, Cistaro A, Filippi M (2014) Neuroimaging in amyotrophic lateral sclerosis: Insights into structural and functional changes. Lancet Neurol 13, 1228-1240.

[26] Tsujimoto M, Senda J, Ishihara T, Niimi Y, Kawai Y, Atsuta N, Watanabe H, Tanaka F, Naganawa S, Sobue G (2011) Behavioral changes in early ALS correlate with voxel-based morphometry and diffusion tensor imaging. J Neurol Sci 307, 34-40.

[27] Rainero I, Rubino E, D'Agata F, Rossetti S, Gastaldi L, Bergui M, Orsi L, Pinessi L, Isaia G, Di Stefano (2014) Voxel-based morphometry reveals gray matter loss in patients with paget's disease of the bone carrying SQSTM1 gene mutations. Neurology 82 (Meeting Abstracts), P6.222.

[28] Whitwell JL, Jack CR Jr, Boeve BF, Senjem ML, Baker M, Ivnik RJ, Knopman DS, Wszolek ZK, Petersen RC, Rademakers R, Josephs KA (2009) Atrophy patterns in IVS10+16, IVS10+3, N279K, S305N, P301L, and V337M MAPT mutations. Neurology 72, 813-820.

[29] Rohrer JD, Ridgway GR, Modat M, Ourselin S, Mead S, Fox NC, Rossor MN, Warren JD (2010) Distinct profiles of brain atrophy in frontotemporal lobar degeneration caused by progranulin and tau mutations. Neuroimage 53, 10701076.

[30] Whitwell JL, Weigand SD, Boeve BF, Senjem ML, Gunter JL, DeJesus-Hernandez M, Rutherford NJ, Baker M, Knopman DS, Wszolek ZK, Parisi JE, Dickson DW, Petersen RC, Rademakers R, Jack CR Jr, Josephs KA (2012) Neuroimaging signatures of frontotemporal dementia genetics: C9ORF72, tau, progranulin and sporadics. Brain 135, 794806.

[31] Sha SJ, Takada LT, Rankin KP, Yokoyama JS, Rutherford NJ, Fong JC, Khan B, Karydas A, Baker MC, DeJesusHernandez M, Pribadi M, Coppola G, Geschwind DH, Rademakers R, Lee SE, Seeley W, Miller BL, Boxer AL (2012) Frontotemporal dementia due to C9ORF72 mutations: Clinical and imaging features. Neurology 79, 1002-1011. 